\title{
Joining the dots between APOE $\varepsilon 4$ and late-onset Alzheimer disease via integrative genomics
}

A new study involving application of network approaches to transcriptome-wide gene expression data has uncovered molecular players that could underlie the known link between the $\varepsilon 4$ allele of apolipoprotein $\mathrm{E}(A P O E)$ and risk of late-onset Alzheimer disease (LOAD; the sporadic form of the disease). "Our findings provide promising new targets for early therapeutic intervention," says Herve Rhinn, lead author of the study.

Three variants of the APOE gene exist, and have differential effects on LOAD risk. Whereas the rare $\varepsilon 2$ allele is associated with decreased risk of LOAD compared with the common $\varepsilon 3$ allele, the $\varepsilon 4$ allele is associated with a greater than threefold increased risk of LOAD relative to $\varepsilon 3$. Indeed, $A P O E \varepsilon 4$ is the strongest genetic risk factor for LOAD, but the underlying mechanisms are poorly defined.

"For our study, we were motivated in part by the ongoing revolution in genome-wide association studies that has opened exciting new perspectives on sporadic neurodegenerative disorders and reaffirmed interest in working as closely as possible to the human genetic context in which these diseases take root," explains Rhinn.

The researchers began by analysing publically available transcriptomewide expression data from 185 cerebral cortex samples taken at autopsy. 215 of the 8,499 identified transcripts showed significant alteration of expression in the same direction in association with LOAD or APOE $\varepsilon 4$, representing a high degree of correlation. Transcriptomic changes associated with LOAD, but not those associated with APOE \&4, also overlapped substantially with ageingassociated transcriptomic changes, suggesting that independent mechanisms link APOE $\varepsilon 4$ and ageing with LOAD risk.

Previous whole-transcriptome gene expression analyses were able to identify genes associated with LOAD, but could

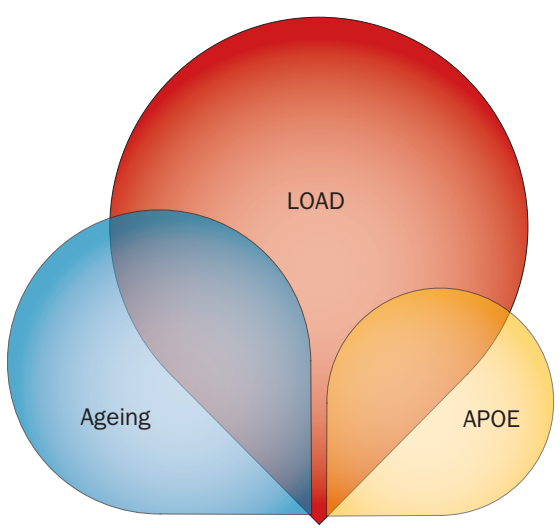

Venn diagram illustrating overlap in transcriptome changes. Original image courtesy of H. Rhinn.

not distinguish causally involved genes from those with a role in processes that are secondary to neuropathology. To overcome such limitations, Rhinn et al. used differential coexpression analysis-a network approach that compares sets of whole-transcriptome data to identify 'master regulator' genes, or causal 'nodes', that are coexpressed with specific gene networks across data sets. "This biostatistical approach appears to be very innovative," says Giulio Maria Pasinetti, Professor of Neurology at Icahn School of Medicine at Mount Sinai, New York, NY, USA, who was not involved in the study.

The 20 most highly ranked candidate genes identified by differential coexpression analysis included several genes previously implicated in cellular processing of amyloid precursor protein (APP) - the source of amyloid- $\beta$ (A $\beta$ ) fibrils that form the brain plaques that are characteristic of Alzheimer disease. Importantly, the study also identified novel molecular mediators of the effect of APOE $\varepsilon 4$ on LOAD: the top two hits in the analysis were RNF219, variants of which have been linked to alterations in lipid metabolism, and $S V 2 A$, a regulator of neuronal endocytosis.

To further probe the mechanism linking the novel candidate genes with APOE \&4 and LOAD, Rhinn et al. employed an in vitro model system comprising mouse neuroblastoma N2a cells that overexpress human APP and increase production of $A \beta$ in response to application of recombinant Apo-E4 protein. RNA knockdown of either RNF219 or SV2A suppressed the amyloidogenic effects of Apo-E4, suggesting that the candidate genes might increase LOAD risk by mediating increased production of $A \beta$ in APOE \&4 carriers.

Next, the investigators applied the antiepileptic drug levetiracetam, which is a selective inhibitor of SV2A, to cultures of neurons induced from human skin fibroblasts from $A P O E \varepsilon 4$ carriers. The drug attenuated the high production of $\mathrm{A} \beta$ by these cells. However, as Pasinetti cautions, "although interesting, many more studies are necessary to prove these concepts are sound and reproducible."

Finally, analysis of PET scans in 206 unaffected elderly individuals revealed a link between brain amyloid load and RNF219 genotype, providing additional support for the computational and in vitro findings.

"This study might provide new information to explore the novel potential 'genomic hit' that could be the basis of agerelated onset of LOAD cases, which are currently termed 'sporadic' cases," notes Pasinetti. Rhinn and colleagues now "aim to connect the dots between APOE $\varepsilon 4$, novel genes such as RNF219 and SV2A, and alterations in amyloid processing." They also intend to apply the same approach to other neurological diseases.

Katie Kingwell

Original article Rhinn, H. et al. Integrative genomics identifies APOE $\varepsilon 4$ effectors in Alzheimer's disease. Nature doi:10.1038/nature 12415

Further reading Liu, C.-C. et al. Apolipoprotein E and Alzheimer disease: risk, mechanisms and therapy. Nat. Rev. Neurol. 9, 106-118 (2013) 